\title{
A Content Analysis of Social and Affect Studies in CSCL
}

\author{
Xiaodan Zhou*, LingHsiu Chen \\ School of Computer and Information Engineering, Xiamen University of Technology, 600, Ligong Rd., \\ Jimei District, Xiamen, 361024, China \\ Department of Information System, Chaoyang University of Technology, 168, Jifeng E. Rd., \\ Wufeng District, Taichung, 41349, Taiwan \\ xdzhou@xmut.edu.cn, ling@cyut.edu.tw
}

\begin{abstract}
Over recent years, while studies about computer-supported collaborative learning increased gradually, more and more researchers recognized the importance of social interactions in the CSCL environments. Social interactions can be divided into on-task and non-task interactions. Non-task interactions embraces some social and affective activities which are not directly learning related such as greetings, social support, and friend making. Many studies have demonstrated that non-task interactions can improve students' learning performance. To investigate the research status of interaction in computer-supported collaborative learning environment, we therefore conduct a research about publication patterns about the social and affective studies from 2001 to 2011.

Index Terms - Collaborative learning, Computer-supported collaborative learning, Non-task interactions, Social and affective issues
\end{abstract}

\section{Introduction}

Social constructivism suggests that knowledge is constructed in a social context at first and is then taken up by individuals, facilitating consistent social interaction among students and their instructor could lead to effective and successful learning [2]. Over recent years, while studies about Computer-Supported Collaborative Learning (CSCL) increased gradually, more and more researchers recognized the importance of social interactions in the CSCL environments. Mcneil et al. categorized students' social interactions in CSCL environments as on-task and non-task interactions[5]. The former includes instructional and learning activities such as group learning and group processing, whereas the latter embraces social and affective activities which are not directly learning related such as greetings, social support, and friend making. Kreijns, Kirschner and Jochems discussed students' social interaction from two dimensions, one dimension is cognitive and educational, the other is social and affective[4]. Social performance encompasses variables like the degree of established social space, sense of community, and degree of trust. social performance can reinforce the cognitive process and then further reinforce the learning performance. Over past years cognitive issues in education and CSCL have already attracted a great deal of attention, so more and more researchers put their effort on the studies of social and affective issues in CSCL.

\section{Research Question}

The purpose of the paper was to investigate the research trends in the field of social and affective issues in computer supported collaborative learning and to explore what categories the social and affective studies can be divided into. After reading abstract and introduction, all collected social and affective studies were classified into several categories and then analyzed by publication year, journal, and category. The results of the analysis might outline the research trends and patterns of the articles related to social and affective issues in CSCL in recent years. It was expected that the results of this study could provide both experienced and novice educators and researchers in the fields of sociability, education, and learning technology with some insightful ideas about the publishing trends of research studies in major journals. The research questions addressed in this paper were listed below:

1) How many topics these articles can be divided into?

2) What are the publishing patterns of the social and affective articles in CSCL from 2001 to 2011 ?

3) What is the trend of published articles about social and affective issues in CSCL in recent years.

\section{Research method and Publication Patterns}

\subsection{Paper selection}

This study used papers published by four major educational journals from 2001 to 2011 as the research sample to examine the research trends in the study of social and affective issues in CSCL. The four journals are: Computers and Education(C\&E), Computers in Human Behavior (CIHR), Learning and Instruction (L\&I) and International Journal of Human-Computer Studies(IJHCS). We have also searched several other journals such as Expert Systems with Applications (ESA), Education Research Review (ERR), Journal of Computer Assisted Learning (JCAL), but no related articles that concentrate on social and affective aspects in CSCL were identified.

This study explored the social and affective issues from an individual perspective, and the selection of articles was processed by a doctoral researcher in educational technology and further validated by a professor in the field. The whole process went through three phases. In the first phase, two hundred and thirty-four articles were selected from seven representative journals using the keyword CSCL and collaborative learning. In the second phase, based on the qualitative analysis of 234 articles' abstract and outline, 20 articles were picked out as social and affective issues. In the final phase, after careful complete article analysis, 16 articles were identified as our study objects. 


\subsection{Research topic analysis}

Based on the methodology of content analysis, the social and affective issues in computer-supported collaborative learning field were categorized into six tentative categories: "Sociability", "Social capital"," Social effect", "Motivation", "Attitude", "Emotion". Definition of social and affective concept in psychology, experts' opinions and article keywords in related journals were all used as references in developing tentative categories. During the data analysis process, these categories were further refined continually by using the constant-comparative method. In the end, five categories were confirmed by classifying "Social effect" into "Social capital" after all articles were reviewed in detail and the final categories are listed below:

1) Sociability. Articles in this research topic include the evaluation of social interaction in CSCL, measurement development about social space, sociability, also include articles explore the factors that affect sociability.
2) Social capital. This category includes studies that investigate social gains during collaborative learning, such as social self-efficacy, trust, social ties etc., which are the opposite of cognitive gains.

3) Motivation. Articles in this research topic explore the influence of learners' motivation on learning activities, knowledge acquisition or knowledge sharing in CSCL environment.

4) Attitude. This category includes articles in which independent variable is culture or the dependent variable in CSCL is satisfaction.

5) Emotion. Emotions are intense feelings that are directed at someone or something, which influences the learning situation in both motivational phase and volitional phase during collaborative learning.

The analysis or coding of these articles was processed by one doctoral researcher and was further validated by a professor.

TABLE 1 Publishing Patterns by Category, Journal and Year

\begin{tabular}{|c|c|c|c|c|c|c|c|c|}
\hline Yategory & 2003 & 2004 & 2005 & 2007 & 2009 & 2010 & 2011 & Total \\
\hline Sociability & $1(\mathrm{C} \& \mathrm{E})$ & $1(\mathrm{CIHB})$ & & $1(\mathrm{C} \& \mathrm{E})$ & & & $1(\mathrm{C} \& \mathrm{E})$ & $4(25 \%)$ \\
\hline Social capital & & & & $1(\mathrm{IJHCS})$ & & $1(\mathrm{~L} \& \mathrm{I})$ & & $2(12.5 \%)$ \\
\hline Motivation & & & $1(\mathrm{C} \& \mathrm{E})$ & & $1(\mathrm{CIHB})$ & & $3(\mathrm{C} \& \mathrm{E}, \mathrm{L} \& \mathrm{I})$ & $5(31 \%)$ \\
\hline Attitude & & $1(\mathrm{IJHCS})$ & & $1(\mathrm{CIHB})$ & & & $2(\mathrm{C} \& \mathrm{E})$ & $4(25 \%)$ \\
\hline Emotion & & & $1(\mathrm{~L} \& \mathrm{I})$ & & & & & $1(6.5 \%)$ \\
\hline Total & 1 & 2 & 2 & 3 & 1 & & 1 & 6 \\
\hline
\end{tabular}

\subsection{Publication patterns}

As we can see from table 1 , the journal Computers \& Education(C\&E) provides a heaviest contribution with total 8 articles. It's publications concentrating on social and affective issues started from 2003 and peaked in 2011(5).The journal Computers in Human Behavior (CIHB) and Learning and Instruction (L\&I) are considered to be the secondary contributor both with 3 articles. Another contributor is International Journal of Human-Computer Studies (IJHCS) with 2 articles. In table 1 , we can seen that "Motivation", "Sociability", and "Attitude" are top three popular research topics from 2001 to 2011 in journals of C\&E,CIHB,IJHCS and L\&I, which is 5(31\%),4(25\%),4(25\%) respectively and will be elaborated in detail in latter sections.

There are two articles belonging to "Social capital" and only one article categorized to "Emotion". During collaborative learning, there are cognitive gains and social gains, such as social self-efficacy, trust, social ties etc. Kenneth Tolmie et al. demonstrated that the social benefits of collaborative learning are a separate outcome of group work, rather than being either a pre-condition for, or a direct consequence of successful activity, but that initial training in group skills may serve to enhance these benefits [1]. Jarvenoja and Jarvela investigated the influence of emotions in CSCL. Their results showed that there were five main sources for students' experienced emotions during the computer-supported inquiry learning: self, context, task, performance, social-driven emotions and volitional emotion control is essential for learning and achievement[3].

\section{Conclusions and Implications}

In this paper, the researchers categorized and analyzed articles related to social and affective issues in CSCL that were published in four representative SSCI journals from 2001 to 2011 ,which is Computers and Education(C\&E), Computers in Human Behavior (CIHR), Learning and Instruction (L\&I) and International Journal of Human-Computer Studies(IJHCS) . Based on the complete article analysis, these articles can be categorized into five sub-categories of "sociability", "social capital", "motivation", "attitude" and "emotion". The publishing patterns by category, journal and year were uncovered. It is obvious that C\&E provides a heaviest contribution with $8(50 \%)$ articles and it's publications started from 2003 and peaked in 2011(5). It also can be seen that "Motivation", "Sociability", and "Attitude" are three most popular research topics from 2001 to 2011 in journals of C\&E,CIHB,IJHCS and L\&I, which is $5(31 \%)$, $4(25 \%), 4(25 \%)$ respectively. "Sociability" studies the CSCL 
system's social capability to facilitate the emergence of a sound social space with attributes as trust and belonging, a strong sense of community, and good working relationships, while other sub-categories mainly focus on the study of characteristic or attribute of learners.

There are some implications of this content review and the results of the analysis provided insights for educators and researchers into research trends and patterns of social and affective issues in CSCL. First, the review of "sociability" articles demonstrated the importance of social interaction and pointed out that Babak's sociability measurement has a good reliability and validity and can be used in future research. Second, the analysis of motivation indicates that although motivation has positive effect on learning activities in individual phase, but it is not significant in collaborative phase, for the context gets more sophisticated or more variables add in. Finally, the review of "attitude" articles indicates that we can't ignore teacher's effect. Teachers can greatly enhance learners' satisfaction in collaborative learning by providing quality instructions and specific guidelines on how to regulate the group, integrating many kinds of media or setting tasks requiring high levels of collaboration.

\section{References}

[1] Kenneth Tolmie A., Topping K. J., Christie D., Donaldson C., Howe C., Jessiman E., Livingston K. \& Thurston A, "Social effects of collaborative learning in primary schools", Learning and Instruction, 20,177-191,2010.

[2] Delfino, M., \& Manca, S., "The expression of social presence through the use of figurative language in a web-based learning environment", Computers in Human Behavior, 23(5), 2190-2211, 2007.

[3] Jarvenoja H.\& Jarvela S., "How students describe the sources of their emotional and motivational experiences during the learning process $\mathrm{A}$ qualitative approach", Learning and Instruction, 15, 465-480,2005

[4] Kreijns, K., Kirschner, P. A., \& Jochems, W., "Identifying the pitfalls for social interaction in computer-supported collaborative learning environments: A review of the research", Computers in Human Behavior, 19(3), 335-353, 2003.

[5] Mcneil, S. G., Robin, B. R., \& Miller, R. M., "Facilitating interaction, communication and collaboration in online courses", Computers \& Geosciences, 26, 699-708, 2000. 